\title{
Pro-Flbrotic Phenotype of Human Skin 338 Fibroblasts Induced by Periostin via Modulating TGF- $\beta$ Signaling
}

Miwa Kanaoka, Yukie Yamaguchi, Noriko Komitsu, Kazuhiko Arima, Kenji Izuhara and Michiko Aihara

Department of Environmental Immuno-Dermatology,

Yokohama City University Graduate School of Medicine, Yokohama, Japan

\section{ABSTRACT}

Background Periostin is a matricellular protein, one of a class of extracellular matrix $(E C M)$-related molecules defined by their ability to modulate cell-matrix interaction. We previously reported an elevated level of circulating periostin in patients with systemic sclerosis (SSc) and its association with severity of skin sclerosis.

Objective We examined the role of periostin in transforming growth factor- $\beta$ (TGF- $\beta$ ) signaling mediating fibrosis.

Methods We first determined periostin levels in skin and lung fibroblasts obtained from SSc patients. ECM proteins and pro-fibrotic factors protein levels were further evaluated in periostin-expressing human skin fibroblasts in the presence or absence of TGF- $\beta$. Following TGF- $\beta$ stimulation, the effects of periostin on Smad proteins were also evaluated by immunoblotting and immunofluorescence staining. Finally, Smad 7 protein levels were assessed in fibroblasts under periostin RNA interference.

Results Periostin was more strongly expressed in skin and lung primary SSC fibroblasts than in healthy subjects. Recombinant periostin (rP)-treated fibroblasts and periostin-expressing fibroblasts produced significantly more ECM proteins in the presence of TGF- $\beta$ than control fibroblasts stimulated with TGF- $\beta$ alone. Overexpression of periostin enhanced the induction of Egr-1 and $\alpha$-SMA in the presence of TGF- $\beta$. In addition, R-Smad activity induced by TGF$\beta$ was not significantly affected by periostin, but the level of Smad 7 was reduced in periostin-expressing fibroblasts under TGF- $\beta$ stimulation. On the other hand, the level of Smad 7 was increased in fibroblasts under periostin RNA interference in the presence of TGF- $\beta$.

Conclusion These results suggest that periostin may contribute to fibrosis by enhancing TGF- $\beta$ signaling via Smad 7 inhibition, which leads to further ECM deposition and perios

\section{INTRODUCTION}

Systemic sclerosis (SSc) results in significant morbidity and mortality due to organ fibrosis characterized by the increased deposition of extracellular matrix (ECM).

We previously reported an elevated level of circulating periostin in SSc patients and its association with the severity of skin sclerosis.

Periostin is a matricellular proteins, one of a class of ECM-related molecules defined by their ability to modulate cell-matrix interaction.

Recent studies have revealed that periostin serves as a critical regulator of Pealing, epithelial mesenchymal transition, and fibrosis.

Periostin plays an important role as a co-factor of TGF- $\beta$ in promoting ECM production and differentiation to myofibroblasts.

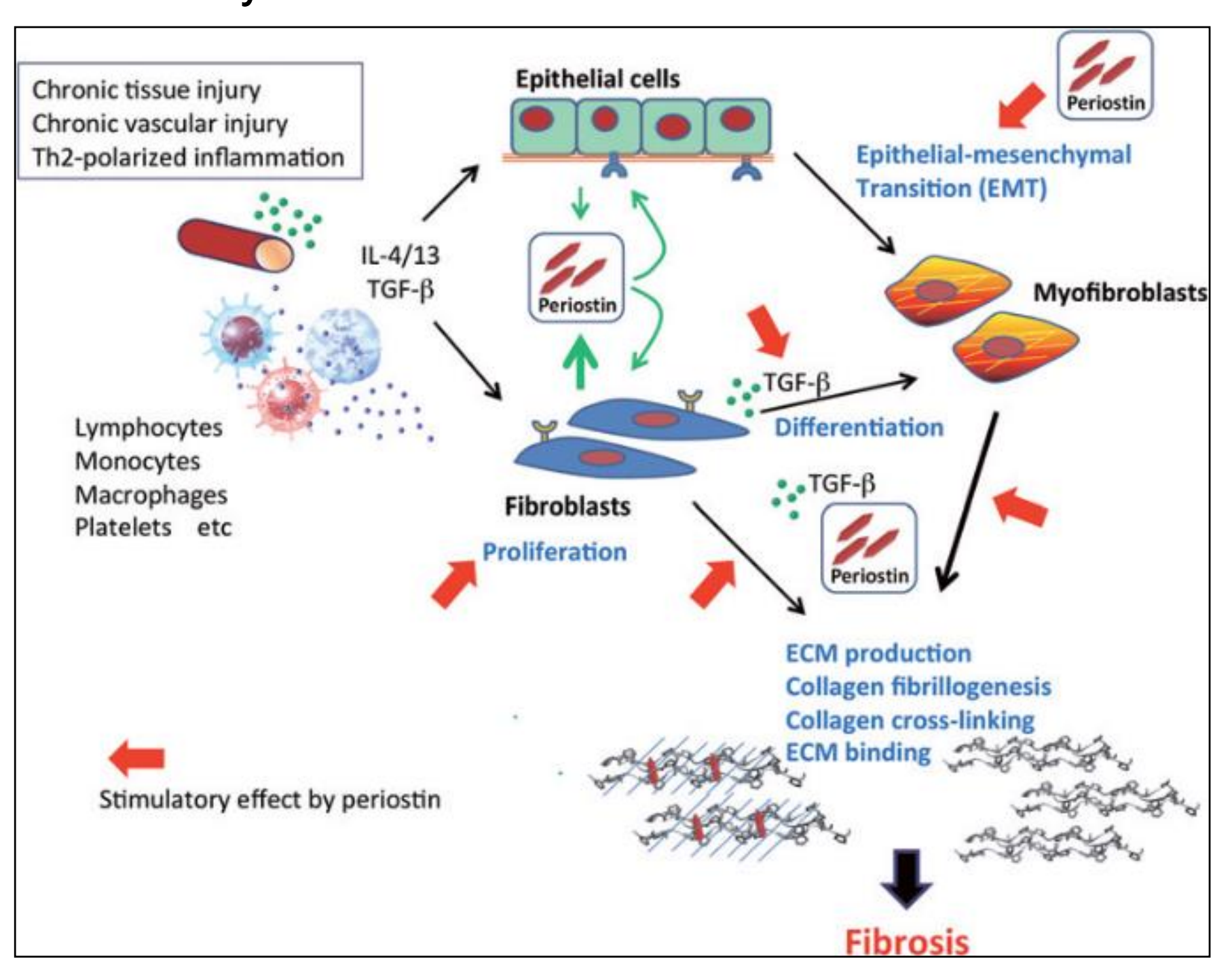

TGF- $\beta$ transduces its signal from the membrane to the nucleus through Smad proteins.

R-Smads are directly phosphorylated to form a complex with Co-Smad and translocate into the cell nuclei, where they regulate gene transcription in co-

Smad 7 down-regulates TGF- $\beta$ signaling

\section{PURPOSE}

To examine the role of periostin in transforming growth factor- $\beta$ (TGF- $\beta$ ) signaling mediating fibrosis.

\section{METHODS \& RESULTS}

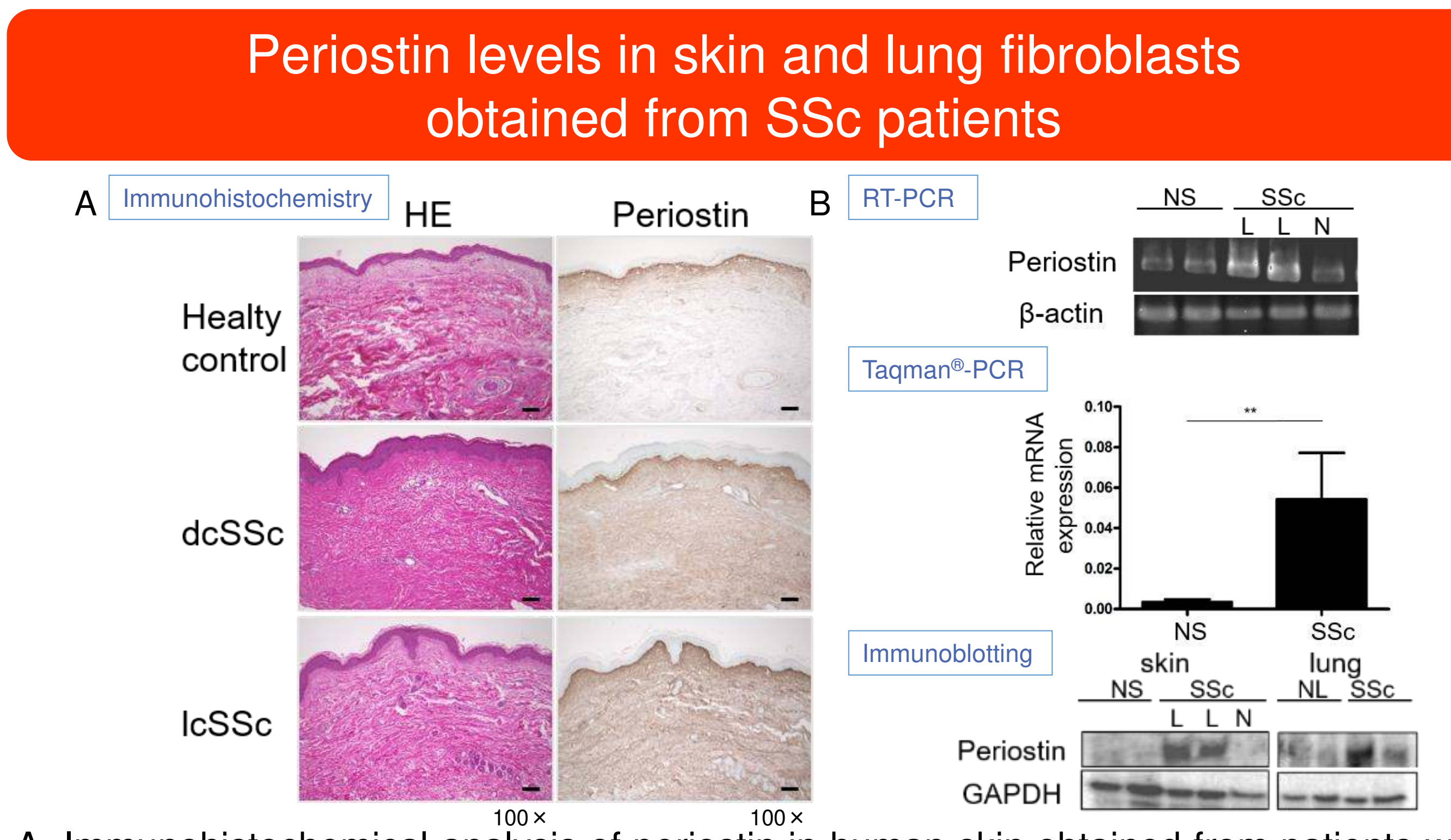

A. Immunohistochemical analysis of periostin in human skin obtained from patients wit systemic sclerosis -difuse culaneous SSC (dCSSc) and limiled cutaneous SSC (ICSSc)- and a healthy control. Haematoxylin-eosin (HE) staining of skin from the same
subjects is also shown. Scale bars $=100 \mu \mathrm{m}$. B. Periostin mRNA and protein levels in normal and SSc fibroblasts -normal skin tibroblasts (NS), scleroderma fibroblasts (SSC), lesional fibroblasts (L), non leasion fbroblasts $(\mathrm{N})$ and normal lung fibroblasts $(\mathrm{NL})-{ }^{*}{ }^{*} \mathrm{p}<0.01$, T-test with Welch's correction, $n=3$.

Periostin was more strongly expressed in skin and lung primary SSc fibroblasts than in
healthy subjects.

ECM production by recombinant periostin in human fibroblasts in the presence or absence of TGF- $\beta$

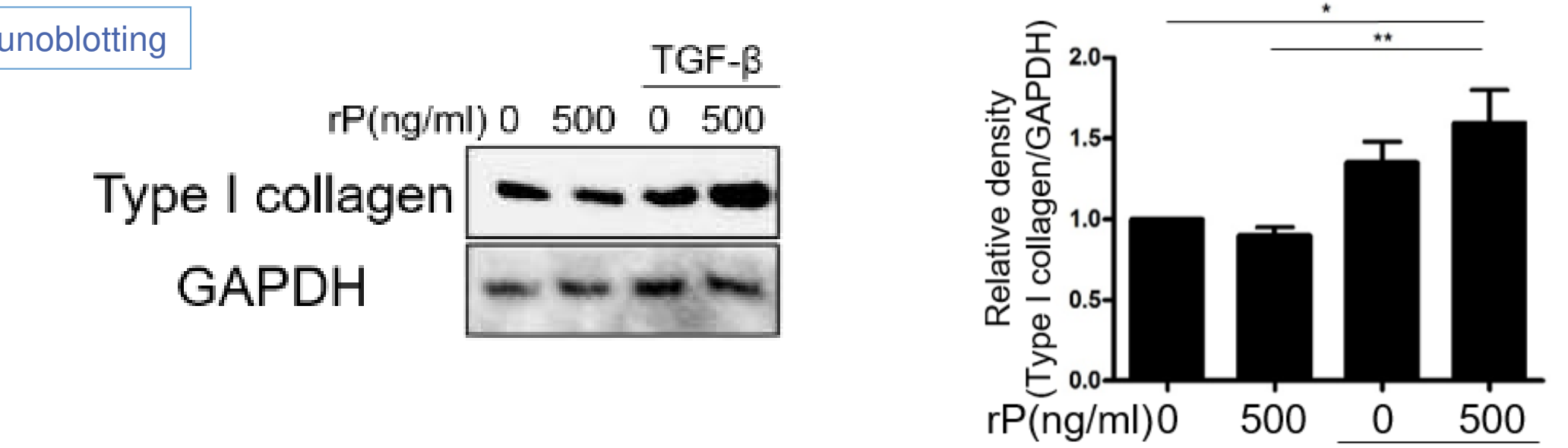

Type I collagen $\alpha 1$ protein levels in supernatants of cultured healthy fibroblasts were confirmed by immunoblotting at $24 \mathrm{~h}$ after stimulation with recombinant periostin ( $\mathrm{rP}$ ) and/or TGF- $\beta .{ }^{*} p<0.05 ;{ }^{* *} p<0.01$, one-way analysis of variance (post-hoc Tukey), $n=4$. Although stimulation with $r P$ alone did not increase type I collagen $p$
stimulation with $r P$ and TGF- $\beta$ enhanced type I collagen production.

\section{ECM production in periostin-expressing fibroblasts} in the presence or absence of TGF- $\beta$

To enhance the function of periostin, a retrovirus expressing human periostin was transfected into human fibroblasts to prepare periostinexpressing fibroblasts.

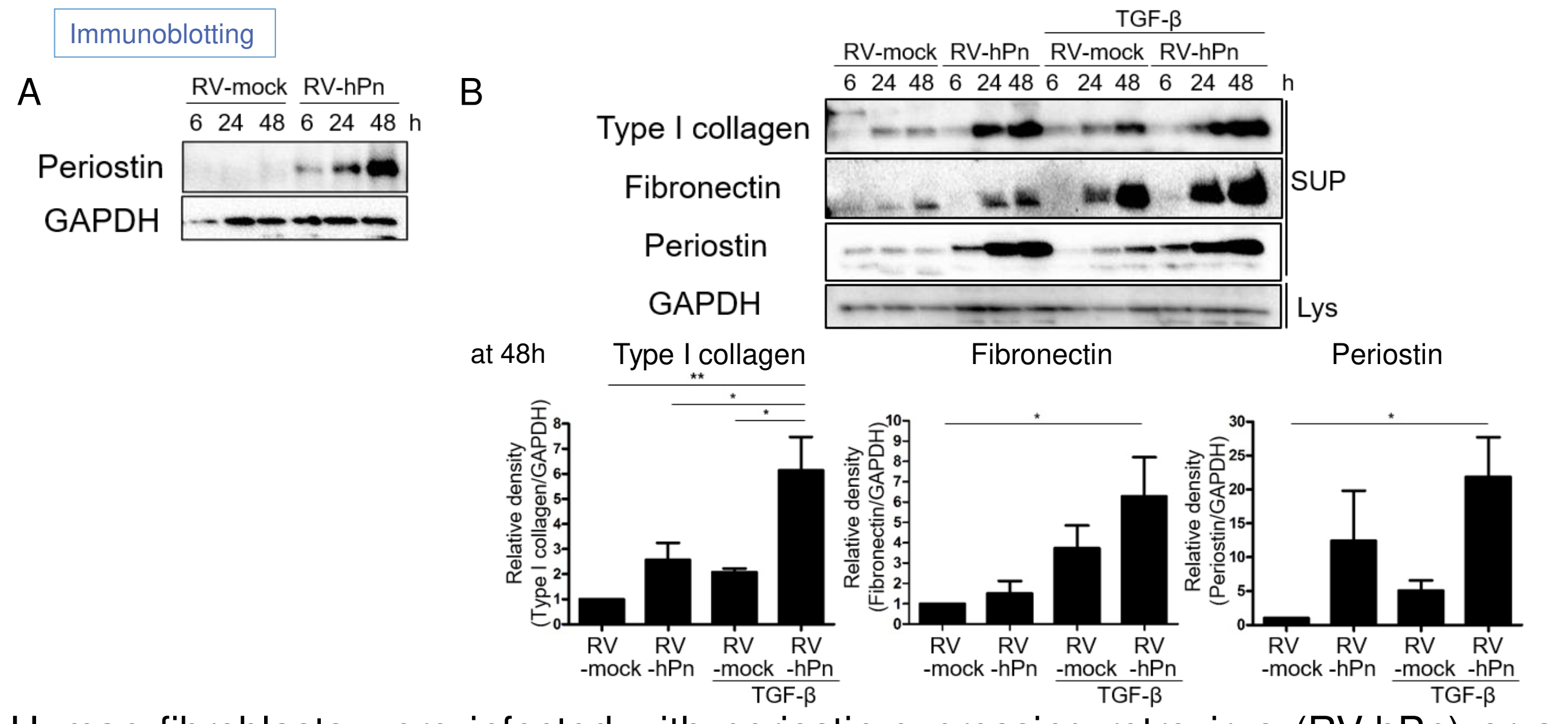

A. Human fibroblasts were in

B. Type I collagen $\alpha 1$ and fibronectin protein levels in supernatants of cultured RV-mock and RV-hPn cells were confirmed by immunoblotting at 6,24 and $48 \mathrm{~h}$ after stimulation with TGF- $\beta$. The levels of each protein at 48 h were quantified using densitometry and expressed as the ratio of each protein to GAPDH. ${ }^{*} \mathrm{p}<0.05 ;{ }^{* *} \mathrm{p}<0.01$; ${ }^{* * *} \mathrm{p}<0.001$ ne-way analysis of variance (post-hoc Tukey), $n=4$.

Pro-fibrotic factors in periostin-expressing fibroblasts in the presence or absence of TGF- $\beta$

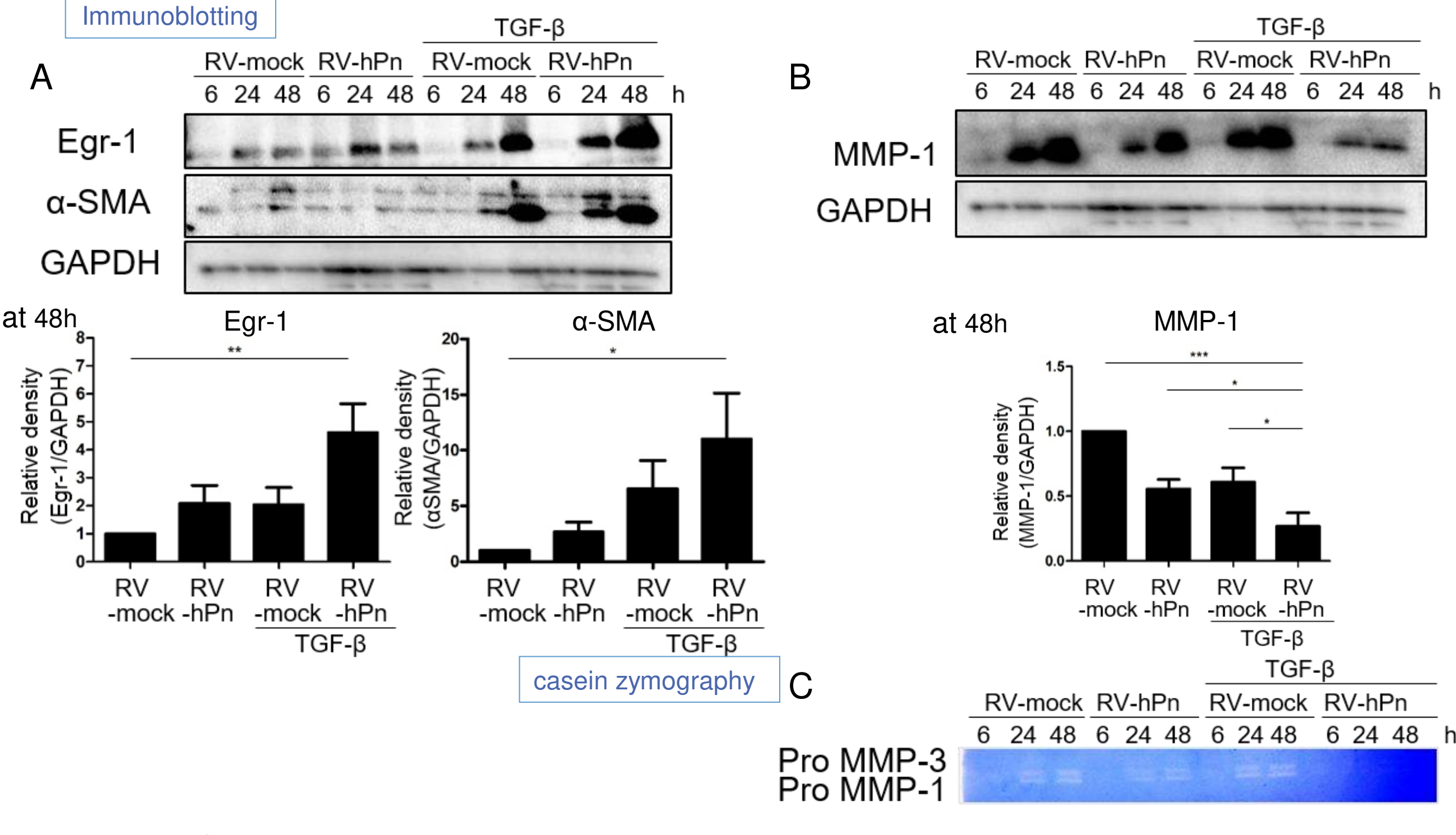

A. Egr-1 and a-SMA protein levels in lysates of cultured RV-mock and RV-hPn cells were confirmed by immunoblotting at 6,24 and $48 \mathrm{~h}$ after stimulation with TGF- $\beta$. The levels of each protein at $48 \mathrm{~h}$ were quantified using densitometry and expressed as the ratio of each protein to GAPDH. ${ }^{*} p<0.05 ;{ }^{* *} p<0.01$, one-way analysis of variance (post-hoc Dunnet) B. Matrix metalloproteinase-1 (MMP-1) protein levels in supernatants of cultured RV-mock and RV-hPn cells were confirmed in the same way. " $p<0.05 ;{ }^{* *} p<0.01$; ${ }^{* * *} p<0.001$, oneway analysis of variance (post-hoc Tukey), $h=4$.

The
R-Smad activity in periostin-expressing fibroblasts in the presence or absence of TGF- $\beta$

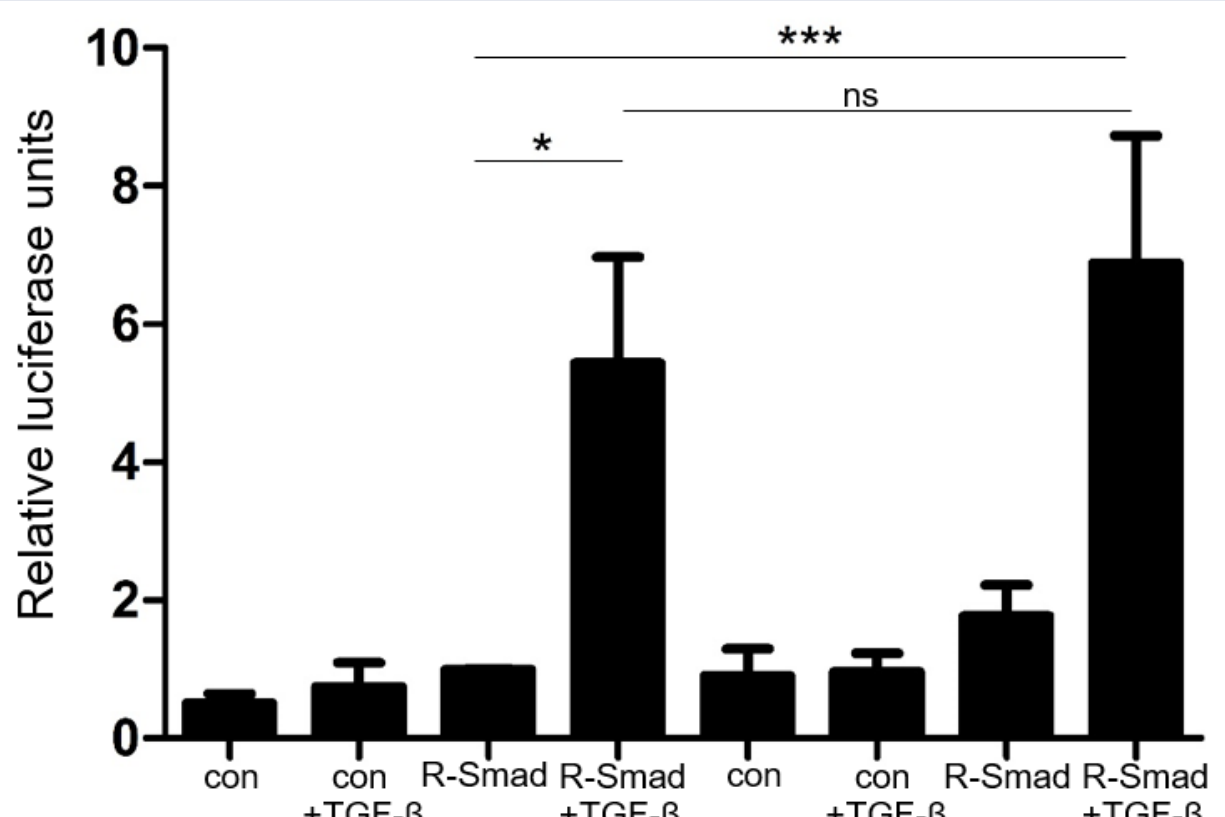

A Smad Reporter Assay was performed on the RV-mock or RV-hPn cells using a Cigna SMAD Reporter Assay kit ${ }^{\Theta} 24 \mathrm{~h}$ prior to TGF- $\beta$ stimulation and R-Smad activity was examined at 18 h after TGF- $\beta$ stimulation. ${ }^{*} p<0.05$; ${ }^{* \star *} p<0.001$; ns, not significant ext way analysis of variance (post-hoc Dunnet), $n=4$.

Smad 7 induced by TGF- $\beta$ in periostin-expressing fibroblasts
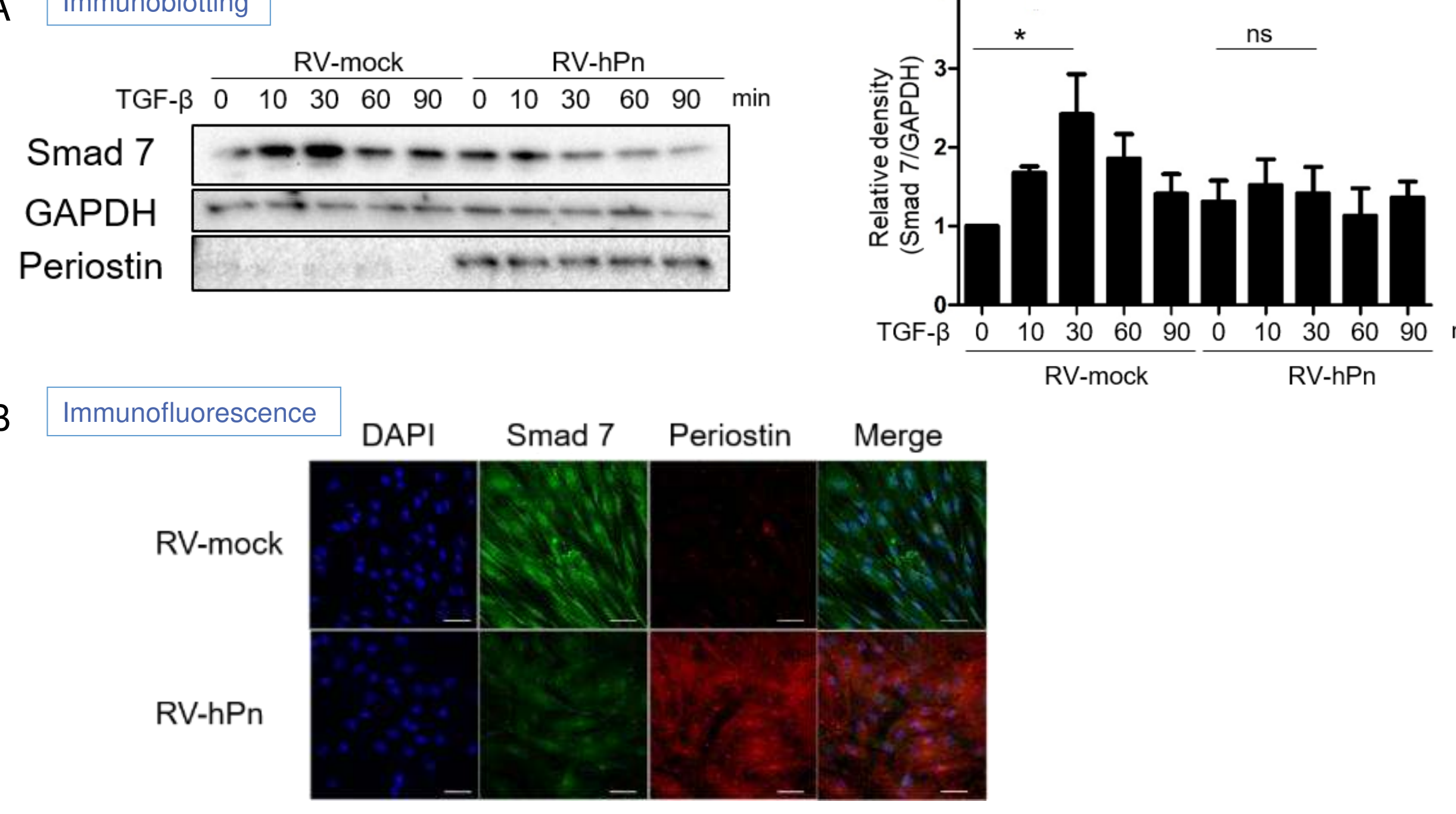

A. Smad 7 protein levels in lysates of cultured PV-hPn and RV-mock cells were . A 30 minues after TGF- $\beta$ stimulation RV-hP and RV-mock cells were stained for Smad 7 and periostin. DAPI was used to identify the nuclei. Scale bars $=100 \mu \mathrm{m}$.

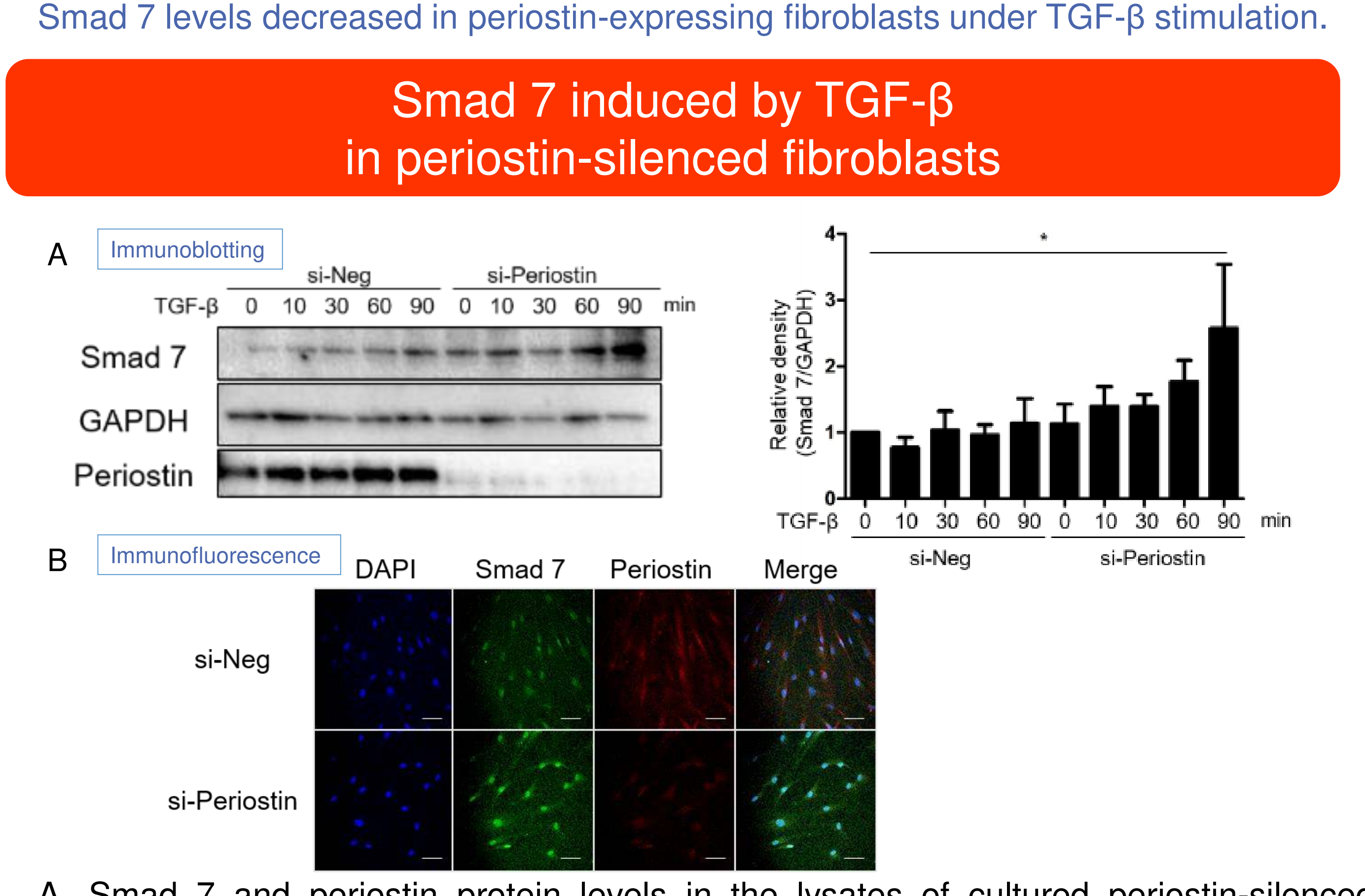

A. Smad 7 and periostin protein levels in the lysates of cultured periostin-silenced 90 minutes after stimulation with TGF- $\beta$. ${ }^{*} p<0.05$, one-way analysis of variance (post90 minutes after
hoc Dunnet), $n=5$.

B. At 30minutes after stimulation of TGF- $\beta$, periostin-silenced fibroblasts and contro fibroblasts were stained for Smad 7 and periostin. DAPI was used to identify the nuclei.

mad 7 levels increased in fibroblasts under periostin RNA intererence in the presence

\section{SUMMARY}

\section{healthy subjects.}

Overexpression of periostin enhanced the induction of ECM production and pro-fibrotic factors in the presence of TGF- $\beta$.

Levels of Smad 7, a down-regulator of TGF- $\beta$ signaling, were reduced in periostin-expressing fibroblasts and increased in fibroblasts with periostin RNA interference.

\section{CONCLUSION}

These results suggest that periostin may contribute to fibrosis by enhancing TGF- $\beta$ signaling via Smad 7 inhibition, which leads to further ECM deposition and periostin generation. Periostin may be a therapeutic target molecule in fibrosis. 